\title{
Molecular epidemiological study of clinical Acinetobacter baumannii isolates: phenotype switching of antibiotic resistance
}

\author{
Chang-Hua Chen ${ }^{1,2}$ and Chieh-Chen Huang ${ }^{3 *}$
}

\begin{abstract}
Background: The presence of clinical Acinetobacter baumannii (A. baumannii) isolates with differing antibiotic resistance phenotypes in the same patient causes difficulties and confusion in treatment. This phenomenon may be caused by reasons such as cross-infection from neighboring patients that switches to different A. baumannii strain, natural mutation of $A$. baumannii, inducing of different antibiotic resistance genes expression or acquisition of genes conferring resistance from another source. To elucidate this question, clinical A. baumannii strains, isolated from the same individual patients, showed antibiotic resistance phenotypes switching during the same hospitalization period, were attentively collected for further analysis. Molecular approaches for phylogenetic analysis, including pulsed field gel electrophoresis, multilocus sequence typing, and short tandem repeat analysis, were employed for the chronological studies.
\end{abstract}

Findings: Our results showed that antibiotic resistance phenotype switching could have occurred as a result through both cross-infection and natural mutation roots. Our results also suggest that rapid phenotype switching between paired isolates could occur during one single course of antibiotic treatment.

Conclusions: Though cross infection caused antibiotic resistance phenotype switching does occur, natural mutation of A. baumannii isolates is particularly cautious for antibiotic treatment.

Keywords: Acinetobacter baumannii, Pulsed field gel electrophoresis, Multilocus sequence typing, Short tandem repeat, Phenotype switch

\section{Findings Introduction}

Acinetobacter baumannii (A. baumannii) was identified from the environment in the early twentieth century, and has been isolated worldwide. The rapid spread of multidrugresistant A. baumannii (MDRAB) in clinical settings has made choosing an appropriate antibiotic to treat these infections difficult for clinicians. A. baumannii within genetically uniform populations exhibit significant phenotypic variability [1]. For example, antibiotic susceptible clinical $A$. baumannii isolates can develop antibiotic resistant phenotypes, in a process called phenotype switching. Such phenotype switching can be perplexing for clinicians, in both interpreting microbiological results and choosing effective antibiotics.

Shanley et al. showed that Acinetobacter calcoaceticus can naturally uptake, incorporate, and stably maintain DNA in vitro [2]. Only a few reports have mentioned the rapid adaptation of $A$. baumannii isolates in a hospital environment $[3,4]$. Determining whether the multiple resistance phenotype switching is due to cross-infection from neighboring patients or from natural mutation of the same A. baumannii isolate is important because of the different strategies needed to resolve the clinical issues. Here we report the rapid change of resistance phenotype of clinical A. baumannii isolates from individual patients during the same admission at a single medical institution in Taiwan.

\footnotetext{
* Correspondence: cchuang@dragon.nchu.edu.tw

${ }^{3}$ Department of Life Science, College of Life Science, National Chung Hsing

University, No. 250 Kuo-Kuang Road, Taichung 402, Taiwan, R.O.C

Full list of author information is available at the end of the article
} 


\section{Material and methods Isolates and phenotyping}

We designed a chronological study to collect pairs of phenotypically-identified $A$. baumannii isolates from individual patients during the same hospitalization period at Changhua Christian Hospital $(\mathrm{CCH})$. Pool of samples for further analysis was collected from January 11998 to December 31 2008. Among those samples, there were three pairs of clinical A. baumannii isolates from $\mathrm{CCH}$ that met the inclusion criteria: Pair 1 (isolates 29-4 and 29-43, numbered according to their position in the $\mathrm{CCH}$ Bacterial Bank), Pair 2 (isolates 10-18 and 10-10), and Pair 3 (isolates 14-91 and 14-81). Phynotypic method to identify those A. baumannii isolates is using a Vitek-2 System (BioMerieux, Marcy l'Etoile, France). And, the isolates were identified according to $16 \mathrm{~S}$ ribosomal RNA region at the molecular level, as previously described [5].

\section{DNA isolation, ribotyping, and detection of short tandem repeats (STR) from clinical A. baumannii isolates}

Genomic DNA was isolated from three colonies from an overnight culture grown on blood agar plates (bioMérieux, Den Bosch, The Netherlands) using a Bacterial Genomic DNA Isolation Kit III according to the manufacturer's instructions (Roche, Mannheim, Germany). The ribotype pattern was interpreted to identify the group to which each strain belonged, as previously described [6]. The primer pair REP1R-I (5-IIICGICGICATCIGGC-3) and REP2-I (5-ICGICTTATCIGGCCTAC-3) [7] was used to amplify putative $R E P$-like elements from the bacterial DNA.

\section{Pulsed field gel electrophoresis}

We followed a standard protocol for pulsed-field gel electrophoresis (PFGE) analysis of the A. baumannii isolates. In brief, A. baumannii were plated on blood agar and incubated in a $5 \% \mathrm{CO}_{2}$ atmosphere at $35^{\circ} \mathrm{C}$ for $16-$ $24 \mathrm{~h}$. Plug slices were digested with $20 \mathrm{U}$ of SgrAI. The DNA fragments were then separated in 1\% Seakem Gold agarose gels (FMC BioProducts) at $14^{\circ} \mathrm{C}$ using a Bio-Rad CHEF DRIII PFGE system (Bio-Rad Laboratories, Hercules, CA, USA). Gels were run in $0.5 \times$ Tris-borate-EDTA (TBE; $\mathrm{pH} 8)$ at a $120^{\circ}$ fixed angle and a fixed voltage $(6 \mathrm{~V} / \mathrm{cm})$, with pulse intervals from 4-40 s for $20 \mathrm{~h}$. Following staining and imaging, the chromosomal DNA restriction patterns produced by PFGE were interpreted using Tenover's categorization [8].

\section{Multilocus sequence typing}

Multilocus sequence typing (MLST) was performed according to the method of Bartual et al. [9]. In brief, housekeeping genes for MLST were selected based on their sequence availability in GenBank, on prior studies of the phylogenetic relationships for the genus Acinetobacter, and on their use in MLST schemes for other bacterial species [1,10-12]. PCR primers were chosen from previous studies or were newly designed for amplification of the seven selected genes: citrate synthase ( $g l t A)$, DNA gyrase subunit B $($ gyr B $)$, glucose dehydrogenase B $(g d h B)$, homologous recombination factor $(\mathrm{rec} A), 60 \mathrm{kDa}$ chaperonin (cpn60), glucose-6-phosphate isomerase ( $g p i)$, RNA polymerase 70 factor $(r p o D)$. All PCR amplifications were performed in a MasterCycler gradient instrument (Eppendorf, Hamburg, Germany). Sequencing of internal fragments ( 450 bp in size) of the selected housekeeping genes was performed in an ABI Prism 377 sequencer using the ABI Prism BigDye terminator cycle sequencing ready reaction kit v. 2 (Applied Biosystems, Foster City, CA, USA) according to the manufacturer's recommendations.

\section{Results}

We collected clinical and microbiological profiles focusing on the three pairs of $A$. baumannii isolates from patients during an individual hospitalization. All three patients stayed at our institute for at least two weeks, and all of them received antibiotics following identification of the A. baumannii isolates (Table 1). The antibiotic susceptibility of clinical A. baumannii isolates are listed in Table 2. Four PFGE fingerprint patterns were detected in the three pairs of $A$. baumannii isolates in Figure 1. Furthermore, there appears to be a clear link of cross-infection between the PFGE types and the clinical data available for the isolates. Interpretation of the MLST data revealed that more than half of the MLST allelic profiles from the three pairs of $A$. baumannii isolates differed from those already in A. baumannii MLST databases (http://pubmlst.org/abaumannii/) [13]. Comparison of the sequence types (ST) of the three paired $A$. baumannii isolates showed similarity between the 29-4 and 29-43 A. baumannii isolates, especially in the allelic profiles of $g l t A, g d h B, \operatorname{rec} A$, and $r p o D$ (Tables 3, 4). However, there was a difference between the 14-91 and 14-81 paired isolates, especially in the allelic profiles of recA, cpn60, and rpoD (Tables 3, 4). These results indicated that isolates 29-4 and 29-43 are the same isolate, and that both paired 14-91 and 14-81 isolates and paired 10-18 and 10-10 isoaltes are different isolates (Tables 3, 4). The fingerprint patterns of the STRs were quite varied (Tables 3,4$)$. It is particularly interesting that rapid phenotype switching between the paired isolates (29-4 and 2943 ) could occur during one course of antibiotic treatment.

\section{Discussion}

The is the first report of phenotype switching of antibiotic resistance in clinical $A$. baumannii isolates in individual patients during the same hospitalization in Taiwan. While A. baumannii has been reported previously in Taiwan, and prolonged administration of broad-spectrum antibiotics 
Table 1 The time line of antimicrobial agents prescription

\begin{tabular}{|c|c|c|c|c|c|c|}
\hline Event & Date & Patient number one, Pair 1 & Date & Patient number two, Pair 2 & Date & Patient number three, Pair 3 \\
\hline Admission day & July 29 & Admission day & July 2 & Admission day & June 3 & Admission day \\
\hline Antimicrobial agent & July 29 and Aug 4 & Cefuroxime & July 2 and July 19 & Ampicillin-sulbactam & June 3 and June 10 & Ampicillin-sulbactam \\
\hline Isolation day & Aug 2 & Isolates $10-10$ from sputum & July 19 & Isolates 29-43 from sputum & June 7 & Isolates 14-91 from sputum \\
\hline Antimicrobial agent & Aug 4 and Aug 7 & Ceftazidime & July 19 and July 26 & Piperacillin-tazobactam & June 10 and June 17 & Cefotaxime \\
\hline Antimicrobial agent & Aug 7 and Aug 10 & Piperacillin-tazobactam & July 26 and Aug 1 & Levofloxacin & June 17 and June 24 & Piperacillin-tazobactam \\
\hline Antimicrobial agent & Since Aug 10 & Imipenem-cilastatin & Since Aug 1 & Imipenem-cilastatin & Since June 24 & Meropenem \\
\hline Isolation day & Aug 16 & Isolates $10-10$ from abscess & Aug 18 & Isolates 29-4 from tip of central catheter & July 7 & Isolates $14-81$ from sputum \\
\hline
\end{tabular}


Table 2 Antibiotic susceptibility of clinical Acinetobacter baumannii isolates

\begin{tabular}{|c|c|c|c|c|c|c|}
\hline Number of isolate & $10-10$ & $10-18$ & $29-43$ & $29-4$ & $14-91$ & $14-81$ \\
\hline Date of isolation & 2-Aug & 16-Aug & 19-Jul & 18-Aug & 9-Jun & 7-Jul \\
\hline Time of isolation & PM 02:38:55 & PM 03:10:10 & PM 04:21:03 & PM 02:47:06 & AM 09:12:47 & AM 10:18:53 \\
\hline Diagnosis & pneumonia & $\begin{array}{l}\text { Soft tissue } \\
\text { infection }\end{array}$ & pneumonia & $\begin{array}{l}\text { Catheter-related } \\
\text { infection }\end{array}$ & pneumonia & pneumonia \\
\hline Specimens & $\begin{array}{l}\text { Sputum, tracheal } \\
\text { aspirate (suction) }\end{array}$ & abscess & $\begin{array}{l}\text { Sputum, tracheal } \\
\text { aspirate (suction) }\end{array}$ & Tip of central catheter & $\begin{array}{l}\text { Sputum, tracheal } \\
\text { aspirate (suction) }\end{array}$ & $\begin{array}{l}\text { Sputum, tracheal } \\
\text { aspirate (suction) }\end{array}$ \\
\hline Antibiotic & Minimum inhibitory & concentrations & $(\mathrm{ug} / \mathrm{mL})$ & & & \\
\hline$\overline{\mathrm{AN}}$ & 8 & 128 & 8 & 128 & 8 & 64 \\
\hline SAM & 32 & 128 & 32 & 128 & 32 & 128 \\
\hline$\overline{C T Z}$ & 8 & 64 & 8 & 64 & 8 & 32 \\
\hline LVF & 2 & 128 & 2 & 128 & 2 & 64 \\
\hline IMP & 2 & 16 & 2 & 16 & 2 & 16 \\
\hline PIP-TAZ & 8 & 256 & 8 & 256 & 8 & 256 \\
\hline CRO & 8 & 128 & 8 & 128 & 8 & 64 \\
\hline CFP & 8 & 256 & 8 & 256 & 8 & 128 \\
\hline MEP & 4 & 32 & 4 & 32 & 4 & 16 \\
\hline
\end{tabular}

Notes: The susceptibility tests were performed using the Vitek-2 GN card (Biomerieux, Marcy l'Etoile, France). The results were interpreted using the CLSI breakpoints (Clinical and Laboratory Standards Institute Performance Standards for Antimicrobial Susceptibility testing; Twenty-First Information Supplement. CLSI document M100-S21, CLSI, Wayne, PA; 2011).

AN: amikacin; SAM: ampicillin-sulbactam; CTZ: ceftazidime; LVF: levofloxacin; IMP: imipenem-cilastatin; PIP-TAZ: piperacillin-tazobactam; CRO: ceftriaxone; CFP: cefepime; MEP: meropenem; colistin and tigecycline and polymyxin B were not provided by Vitek-2 System.

will induce the development of antibiotic resistance in clinical A. baumannii isolates, little is known about the current clinical situation. It was demonstrated that a important evolutionary change of a single genotype was fundamental to the continuous rise observed in the number of $A$. baumannii infections [4].

The current study suggests that natural transformation and mutation of genotypes occurred in clinical
A. baumannii isolates $29-43$ and $29-4$ on the basis of PFGE. We used three methods to determine the genetic similarity of the paired A. baumannii isolates: PFGE, MLST, and STR. Snelling et al. described a PCR assay using repetitive extragenic palindromic sequences to type A. calcoaceticus and A. baumannii strains [14], while Alcala et al. characterized a meningococcal epidemic wave using a MLST method [15], similar to that used in

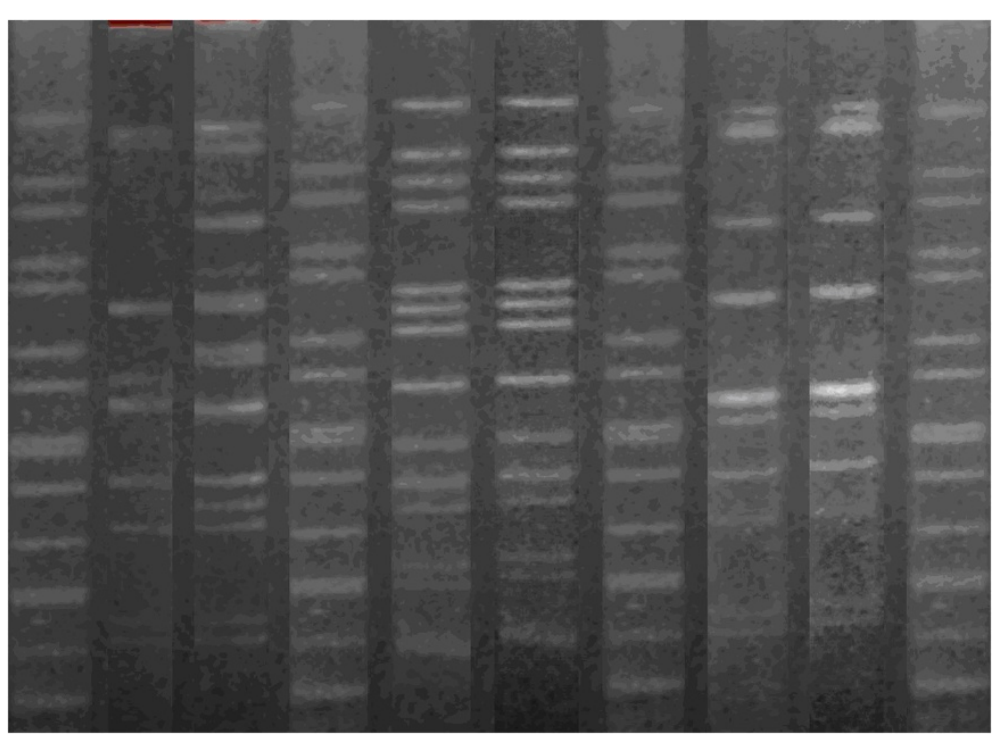

Figure 1 PFGE fingerprints of three pairs of clinical A. baumannii isolates following digestion with the SgrAl restriction enzyme. 
Table 3 The results of pulsed field gel electrophoresis, multilocus sequence typing, and short tandem repeat analysis of three pairs of $A$. baumannii isolates

\begin{tabular}{|c|c|c|c|c|c|c|c|c|c|c|c|}
\hline Isolates & Geno type( $(t)$ & PFGE type & ST Type(‡) & gltA & gyrB & $g d h B$ & $\operatorname{rec} A$ & cpn60 & gpi & $r p o D$ & STR type \\
\hline $10-18$ & $\mathrm{Ab} 1$ & $A$ & this study (a1) & 6 & this study & this study & this study & this study & this study & this study & I \\
\hline $10-10$ & $\mathrm{Ab} 2$ & $A$ & this study (a2) & 11 & this study & this study & this study & this study & this study & this study & $\|$ \\
\hline $29-4$ & $\mathrm{Ab} 3$ & $B$ & this study (a3) & 1 & this study & 3 & 2 & 2 & this study & 3 & III \\
\hline $29-43$ & $\mathrm{Ab} 3$ & $B$ & this study (a4) & 1 & 3 & 3 & 2 & this study & 7 & 3 & IX \\
\hline $14-91$ & $\mathrm{Ab} 4$ & $C$ & this study (a5) & 1 & this study & this study & 2 & 1 & 23 & 18 & $x$ \\
\hline $14-81$ & $\mathrm{Ab} 5$ & $D$ & this study (a6) & 1 & this study & 3 & 2 & 2 & this study & 3 & $X I$ \\
\hline
\end{tabular}

Sequences of amplified genes were compared with sequences from the A. baumannii MLST website (http://pubmlst.org/abaumannii/).

ST: sequence type.

t the given name of the genotype is defined as $A$. baumannii + number (Ab + number).

$\neq$ the given name of the genotype is defined as Acinetobacter isolate + number ( $a+$ number).

our study. The congruence between the MLST, PFGE, and STR data suggests that the findings of the current study are sound; however, further experiments are required to prove the relationships among the paired isolates.

In this study, we discovered natural mutation and rapid change of antibiotic resistance phenotype of clinical A. baumannii isolates from an individual patient. This is alarming as this particular clone seems to be able to effectively fill niches that were essentially uninhabited by $A$. baumannii in the past. Even in a relatively closed environment, the isolates of identical PFGE fingerprint patterns showed a variety of MLST patterns. Apparently, the MLST patterns of paired isolates 29-4 and 29-43 are capable of withstanding background mutation. It is possible that the mutation rate of this particular isolate may contribute to its success in coping with different environments.

\section{Conclusions}

This study provides novel insight into the clinical problem of whether different A. baumannii isolates from the same patient are due to cross-infection from neighboring patients or from natural mutation. This is important for clinicians because the treatments for the two causes are different. The approach for the first phenomenon is to enhance contact precautions in the clinical practice, whereas the second is the stepwise prescription of different antibiotics.

\section{Availability of supporting data}

None.

\section{Ethical approval}

Not required.

Table 4 Aligmnent for three pairs of Acinetobacter baumannii siolates

\begin{tabular}{|c|c|c|c|c|c|c|c|}
\hline $10-10$ VS $10-18$ & gltA & gyrB & gdhB & recA & cpn60 & gpi & rpoD \\
\hline Length & 484 & 927 & 744 & 371 & 454 & 358 & 860 \\
\hline Score & 684 & 1218 & 1258 & 667 & 819 & 662 & 1589 \\
\hline Identities & $446 / 484$ & $835 / 922$ & 699/707 & $361 / 361$ & $446 / 447$ & $358 / 358$ & $860 / 860$ \\
\hline Difference & 38 & 87 & 8 & 0 & 1 & 0 & 0 \\
\hline Gaps & $0 / 484$ & $4 / 922$ & $4 / 707$ & $0 / 361$ & $1 / 447$ & $0 / 358$ & $0 / 860$ \\
\hline 29-4 VS 29-43 & gltA & gyrB & gdhB & recA & cpn60 & gpi & rpoD \\
\hline Length & 484 & 936 & 396 & 371 & 454 & 363 & 513 \\
\hline Score & 894 & 806 & 732 & 686 & 778 & 464 & 948 \\
\hline Identities & $484 / 484$ & $450 / 457$ & $396 / 396$ & $371 / 371$ & $421 / 421$ & $265 / 272$ & $513 / 513$ \\
\hline Difference & 0 & 7 & 0 & 0 & 0 & 7 & 0 \\
\hline Gaps & 0 & 0 & 0 & 0 & 0 & 0 & 0 \\
\hline $14-91$ VS 14-81 & gltA & gyrB & gdhB & recA & cpn60 & gpi & rpoD \\
\hline Length & 484 & 932 & 396 & 371 & 421 & 360 & 513 \\
\hline Score & 894 & 1701 & 399 & 686 & 773 & 392 & 931 \\
\hline Identities & $484 / 484$ & $924 / 925$ & $318 / 369$ & $371 / 371$ & $420 / 421$ & $253 / 273$ & $510 / 513$ \\
\hline Difference & 0 & 1 & 51 & 0 & 1 & 20 & 3 \\
\hline Gaps & 0 & 1 & 0 & 0 & 0 & 2 & 0 \\
\hline
\end{tabular}




\section{Abbreviations}

A. baumannii: Acinetobacter baumannii; CCH: Changhua christian hospital; cpn60: 60 kDa chaperonin; gdhB: Glucose dehydrogenase B; gltA: Citrate synthase; gpi: Glucose-6-phosphate isomerase; gyrB: DNA gyrase subunit B; MDRAB: Multidrug-resistant Acinetobacter baumannii; MLST: Multilocus sequence typing: PFGE: pulsed-field gel electrophoresis; recA: Homologous recombination factor; rpoD: RNA polymerase 70 factor; ST: Sequence types; STR: Short tandem repeats.

\section{Competing interests}

Both authors declare that they have no competing interests.

\section{Authors' contributions}

$\mathrm{CHC}$ and $\mathrm{CCH}$ designed and performed this study. $\mathrm{CCH}$ analyzed the data regarding the infectious diseases and wrote the manuscript. Both authors read and approved the final manuscript.

\section{Acknowledgements}

Authors gratefully acknowledge the help of Dr. Chien-Shun Chiou at Center for Disease Control, Taiwan for invaluable assistance with the development of the array-based MLST system. Authors also thank Hsusan-Pei Lin, and Chialin Chang for technical assistance.

\section{Author details}

'Division of Infectious Diseases, Department of Internal Medicine, Changhua Christian Hospital, No. 135 Nanshiao street, Changhua 500, Taiwan, R.O.C. 2Department of Nursing, College of Medicine \& Nursing, Hung Kuang University, No. 1018, Sec. 6, Taiwan Boulevard, Shalu District, Taichung City 43302, Taiwan, R.O.C. ${ }^{3}$ Department of Life Science, College of Life Science, National Chung Hsing University, No. 250 Kuo-Kuang Road, Taichung 402, Taiwan, R.O.C.

Received: 7 June 2013 Accepted: 11 August 2013

Published: 21 August 2013

\section{References}

1. Ko E, Yomo T, Urabe I: Dynamic clustering of bacterial population. Physica D 1994, 75:81-88

2. Shanley MS, Ahmadian-Tehrani M, Benjamin RC, Leher HF: Natural transformation in Acinetobacter calcoaceticus. SAAS Bull Biochem Biotechnol 1990, 3:27-31.

3. Nwugo CC, Arivett BA, Zimbler DL, Gaddy JA, Richards AM, Actis LA: Effect of Ethanol on Differential Protein Production and Expression of Potential Virulence Functions in the Opportunistic Pathogen Acinetobacter baumannii. PLoS One 2012, 7:e51936.

4. Zander E, Chmielarczyk A, Heczko P, Seifert H, Higgins PG: Conversion of OXA-66 into OXA-82 in clinical Acinetobacter baumannii isolates and association with altered carbapenem susceptibility. J Antimicrob Chemother 2013, 68:308-311.

5. Sahl JW, Johnson JK, Harris AD, Phillippy AM, Hsiao WW, Thom KA, Rasko DA: Genomic comparison of multi-drug resistant invasive and colonizing Acinetobacter baumannii isolated from diverse human body sites reveals genomic plasticity. BMC Genomics 2011, 12:291.

6. Gerner-Smidt P: Ribotyping of the Acinetobacter calcoaceticus-Acinetobacter baumannii complex. J Clin Microbiol 1992, 30:2680-2685.

7. Versalovic J, Koeuth T, Lupski JR: Distribution of repetitive DNA sequences in eubacteria and application to fingerprinting of bacterial genomes. Nucleic Acids Res. 1991, 19:6823-6831.

8. Turnover FC: Plasmid fingerprinting. A tool for bacterial strain identification and surveillance of nosocomial and community-acquired infections. Clin Lab Med 1985, 5:413-436.

9. Bartual SG, Seifert H, Hippler C, Luzon MAD, Wisplinghoff $H$, Rodríguez-Valera F: Development of a Multilocus Sequence Typing Scheme for Characterization of Clinical Isolates of Acinetobacter baumannii. J Clin Microbiol 2005, 43:4382-4390.

10. Hülter $\mathrm{N}$, Wackernagel W: Double illegitimate recombination events integrate DNA segments through two different mechanisms during natural transformation of Acinetobacter baylyi. Mol Microbiol 2008 67:984-995

11. Kotetishvili M, Stine OC, Chen Y, Kreger A, Sulakvelidze A, Sozhamannan S, Morris JG: Multilocus sequence typing has better discriminatory ability for typing Vibrio cholerae than does pulsed-field gel electrophoresis and provides a measure of phylogenetic relatedness. J Clin Microbiol 2003, 41:2191-2196.

12. Maiden MC, Bygraves JA, Feil E, Morelli G, Russell JE, Urwin R, Zhang Q, Zhou J, Zurth K, Caugant DA, Feavers IM, Achtman M, Spratt BG: Multilocus sequence typing: a portable approach to the identification of clones within populations of pathogenic microorganisms. Proc Natl Acad Sci USA 1998, 95:3140-3145.

13. Bartual SG, Seifert H, Hippler C, Luzon MA, Wisplinghoff H, Rodríguez-Valera F: Development of a multilocus sequence typing scheme for characterization of clinical isolates of Acinetobacter baumannii. Clin Microbiol 2005, 43:4382-4390

14. Snelling AM, Gerner-smidt P, Hawkey PM, Heritage J, Parnell P, Porter C, Bodenham AR, Inglis T: Validation of Use of Whole-Cell Repetitive Extragenic Palindromic Sequence-Based PCR (REP-PCR) for Typing Strains Belonging to the Acinetobacter calcoaceticus-Acinetobacter baumannii Complex and Application of the Method to the Investigation of a Hospital Outbreak. J Clinical Microbiology 1996, 34:1193-1202.

15. Alcala B, Salcedo C, Arreaza L, Berro'n S, de la Fuente L, va'Zquezj JA: The epidemic wave of meningococcal disease in Spain in 1996-1997: probably a consequence of strain displacement. Med Microbiol 2002, 51:1102-1106.

doi:10.1186/1476-0711-12-21

Cite this article as: Chen and Huang: Molecular epidemiological study of clinical Acinetobacter baumannii isolates: phenotype switching of antibiotic resistance. Annals of Clinical Microbiology and Antimicrobials 2013 12:21.

\section{Submit your next manuscript to BioMed Central and take full advantage of:}

- Convenient online submission

- Thorough peer review

- No space constraints or color figure charges

- Immediate publication on acceptance

- Inclusion in PubMed, CAS, Scopus and Google Scholar

- Research which is freely available for redistribution 